\title{
Error analysis of delayed response in aged squirrel monkeys
}

\author{
JAMES E. KING and RAENEL R. MICHELS \\ University of Arizona, Tucson, Arizona
}

\begin{abstract}
Young adult and aged squirrel monkeys were tested on variations of a two-choice, spatial delayedresponse task. Aged monkeys committed more errors than did young monkeys. However, the diminished accuracy of the aged monkeys was not attributable to a memory deficiency because the difference was independent of delay interval. Aged monkeys did not display less responsesequence variability and were no more likely to commit systematic errors than were young monkeys. When response accuracy decreased as a result of increased delay intervals, or absence of a predelay cue, both age groups increased the proportion of errors attributable to random responding; however, the proportion of errors attributable to systematic errors either decreased or remained constant.
\end{abstract}

The loss of short-term memory with aging, at one time a controversial hypothesis, has now been conclusively established, although the magnitude of the deficit is highly task-dependent (see Kubanis \& Zornetzer, 1981; Meudell, 1983 , for reviews). Variations of the delayed-response problem have been used in successful demonstrations of age-dependent deterioration of performance by nonhuman primates including rhesus monkeys (Bartus, Fleming \& Johnson, 1978; Medin, 1969), capuchin monkeys (Bartus, Dean, \& Beer, 1980), and chimpanzees (Riopelle \& Rogers, 1965).

A well-known truism about animal learning and memory testing is that response sequences contain a mixture of systematic and unsystematic error-producing sequences (Fobes \& King, 1982; Harlow, 1959; Lentz \& King, 1981; Levine, 1965). The position habit is a wellknown example. A basic question about aging effects on delayed response is whether age affects systematic and nonsystematic error production equally, or whether systematic errors increase disproportionally with age. The gerontological literature contains considerable speculation and some evidence that aged humans and animals have difficulty suppressing a preferred response and are consequently highly susceptible to perseverative errors and proactive inhibition (e.g., Birren, 1962; Elias \& Elias, 1976; Goodrick, 1968, 1972, 1975). Bartus, Dean, and Fleming (1979) observed elevated rates of perseverative errors by aged rhesus monkeys during discriminationreversal problems. However, the animal evidence is not totally consistent, in part, no doubt, because of large differences in the tasks (e.g., Bernstein, 1961).

The question addressed by the experiment described here is whether aged squirrel monkeys are more suscept-

Data reported in this paper were presented at the annual meeting of the American Society of Primatologists in Austin, TX, June 1986. Requests for reprints should be sent to James E. King, Department of Psychology, University of Arizona, Tucson, AZ 85721. ible than young monkeys to perseveration and proactive inhibition from previous delayed-response trials. In order to answer this question, a type of analysis that we have termed sequential state theory (SST) was performed on successive two-trial response sequences to measure the strengths of systematic and unsystematic error-producing responses. Independent variables included delay interval and the presence or absence of raising a Plexiglas screen between the predelay cue and the monkeys' responses.

\section{METHOD}

\section{Subjects}

The aged monkeys were 4 male and 2 female squirrel monkeys (Saimiri sciureus) whose ages were estimated to range from 14 to 20 years on the basis of dentation and arrival dates in our laboratory. The mean longevity of captive squirrel monkeys is about 20 years (Hopf \& Ploog, 1979). One of the aged females died after the completion of Phase 1 . The young monkeys were 5 males and 2 females with ages between 3 and 5 years. No monkeys had served in any previous delayed-response experiments, but 1 aged subject had prior experience learning color discriminations.

\section{Apparatus}

The monkeys were tested in $31 \times 36 \times 38 \mathrm{~cm}$ stainless steel cages used for transportation between the colony and the testing rooms. Removal of the guillotine door of the transport cage gave the monkey access to a $31 \mathrm{~cm}$-square response panel. Three $5 \mathrm{~cm}$ square windows constructed from one-way screen were positioned horizontally across the center of the panel and recessed $2 \mathrm{~cm}$ from the panel's surface. The center window was inoperative. Incandescent lights behind the right and left windows could illuminate small, empty chambers behind the windows, thereby making the chambers visible to the monkey. The recessed walls of the two windows contained photocell units that projected a horizontal infrared beam in front of the windows. Momentary interruption of a beam by the monkey caused a response to be registered. Correct responses elicited a one-sec tone, whereas incorrect responses simply activated a monitor light visible only to the tester. A wooden block with a recessed foodwell on top was located below each window and held in place by magnets. The front surface of the block was flush with the front of the response panel. A transparent Plexiglas 
screen was mounted in front of the response panel and could be manually raised and lowered by the tester.

\section{Procedure}

Phase 1. Each trial was initiated with the Plexiglas screen interposed between the monkey and the response panel. Both window lights were off. Foodwells under both the right and left windows were loaded with bits of marshmallows, raisins, or peanuts, depending upon the individual monkey's preference.

At the start of the trial, one of the two window lights was turned on for $2 \mathrm{sec}$. A predetermined delay interval ensued, after which the tester raised the Plexiglas screen. If the monkey interrupted the photocell beam in front of the previously lighted window, the tone immediately sounded and the tester pushed the reward block under the window toward the monkey, giving it access to the reward. As soon as the monkey retrieved the reward, the tester pulled the tray back to its original position and lowered the Plexiglas screen. If the monkey responded to the incorrect window, the tester immediately lowered the Plexiglas screen. Each trial began $25 \mathrm{sec}$ after the monkey's response on the preceding trial.

Two trials with a 0 -sec delay were presented at the start of each test day. Since the tester raised the Plexiglas screen at the offset of the window light, the actual delay between the window light offset and the availability of the response was slightly more than $0 \mathrm{sec}$. These two initial trials were treated as warm-up trials and were not used in the data analysis. After the two warm-up trials, $\mathbf{4 0}$ trials were presented, equally divided into five conditions. Delays of $0,2,4$, and $8 \mathrm{sec}$ constituted four of those conditions. In addition, a no-cue condition was included that was presented as a 0-sec delay, except that neither window light was turned on at the start of the trial. One side was correct, but since the monkey had no cue to identify it, the no-cue condition necessarily led to a $50 \%$ error rate. The no-cue condition was included to provide levels of systematic and unsystematic response sequences under a condition of total uncertainty.

The presentation order of the five conditions was randomized with the restriction that the same condition not be presented on two successive trials. In addition, each day the sequence of correct rightleft positions was randomized with the following restrictions: (1) no position could be correct on more than three successive trials, (2) each condition would be presented eight times with right correct and eight times with left correct, and (3) for each condition, right-correct and left-correct trials would each be presented an equal number of times preceded by right-correct and by left-correct trials. This extensive balancing was necessary to perform the responsesequence analysis described in the following section. The monkeys were tested 5 days a week for a total of 24 days.

Phase 2. Two new conditions were used in Phase 2. A constant condition was included in which the window light was illuminated at the start of the trial and remained on after the Plexiglas screen was raised $2 \mathrm{sec}$ later. The light was terminated when the monkey either responded to the correct, lighted window or to the incorrect, darkened window. A true 0 -sec delay condition was also included. In this condition, the window light was turned on and the Plexiglas screen was raised simultaneously at the start of the trial. After $2 \mathrm{sec}$, the window light was turned off. Correct or incorrect responses were recorded only for responses that occurred after offset of the window light. Responses that occurred before the light offset had no effect. In fact, responses of this type were very infrequent. Thus, all recorded responses had to occur in the absence of the window light. Also included was an 8-sec delay, presented the same as in Phase 1 . Continuous, true 0 -sec delay and 8-sec delay conditions were presented 18 times each test day for a total of 48 trials. Randomization of the presentation order for conditions and balancing of rewarded position sequences followed the Phase 1 procedures. Testing continued for 12 test days.

\section{Response-Sequence Analysis}

The analysis of response sequences was designated as sequential state theory (SST) and was calculated as if the subject were in one of several possible states on each trial. Each state had an associated type of response that always occurred when the subject was in that state. If the subject was in any other state, the response occurred with probability 0.5 . For example, assume that $A$ and $P(a)$ represent estimates of the probabilities of State $A$ and its associated Response a. Since Response a occurs with probability 1 when the subject is in State $A$ and with probability 0.5 when the subject is in any other state, $P(a)=A+0.5(1-A)$ and $A=2 P(a)-1$. A set of states were defined that described the most important constraints on responding, and observed data were used to estimate the probabilities of the associated responses corresponding to $\mathbf{P}(\mathrm{a})$. A was then easily calculated from the above formula. Data were analyzed as two-trial sequences categorized into a $4 \times 4$ matrix based on whether responses were correct $(+)$ or incorrect $(-)$, and whether they were to the right (r) or left (l) side. Table 1 shows the resulting response-sequence matrix.

State D (Detect) reflects accurate remembrance of the correct side and is associated with a correct $(+)$ response. Therefore, $D=$ $2 \mathrm{P}\left(+_{2}\right)-1$, where $P\left(+_{2}\right)$ is the observed proportion of sequences with a correct response on Trial $-2 . P\left(+_{2}\right)$ is simply the summed frequencies of the first and third rows of the matrix divided by the total number of responses.

A relatively weak but persistent source of errors was the monkeys' tendency to choose the position that was incorrect on the previous trial. This state, designated as $\mathbf{G}$, was defined as a positionbased win-shift/lose-stay (see Levine, 1965), and was associated with a response on Trial 2 to the position that was incorrect on Trial 1. Thus, following a correct response, State $\mathbf{G}$ was manifested by a response to the position not chosen on Trial 1 (shift), and following an incorrect response it was manifested by a response to the position chosen on Trial 1 (stay). A shift response is therefore manifested by either an $r_{2} l_{1}$ or an $l_{2} r_{1}$ sequence, and a stay response by either an $r_{2} r_{1}$ or an $l_{2} l_{1}$ sequence. The observed proportion of win-shift/lose-stay responses therefore had one component based on Trial 2 stay responses $\left(\mathrm{St}_{2}\right)$ and one based on Trial 2 shift responses $\left(\mathrm{Sh}_{2}\right)$. If these two estimates are weighted equally, the estimated proportion of positional win-shift/lose-stay responses $\mathrm{P}(\mathrm{w}-\mathrm{s} ; 1-\mathrm{s})=\left[\mathrm{P}\left(\mathrm{Sh}_{2} \mid+{ }_{1}\right)+\mathrm{P}\left(\mathrm{St}_{2} \mid-{ }_{1}\right)\right] / 2$ and $\mathbf{G}=$ $2 P(w-s ; 1-s)-1$.

Table 1

Response Sequence Matrix for Calculating State Strengths

\begin{tabular}{|c|c|c|c|c|}
\hline & $\mathbf{r}_{1} \mathbf{r}_{2}$ & $r_{1} \mathbf{l}_{2}$ & $l_{1} l_{2}$ & $\mathrm{l}_{1} \mathrm{r}_{2}$ \\
\hline$++_{1}+2$ & & & & \\
\hline$+_{1}-{ }_{2}$ & & & & \\
\hline$-{ }_{1}+2$ & & & & \\
\hline-2 & & & & \\
\hline
\end{tabular}

Formulas Used to Define State Strengths
$D$ (detect)
$=2 P\left(t_{2}\right)-1$
G (positional win-shift/lose-stay)
$=P\left(r_{2} r_{1}\right.$ or $\left.l_{1} l_{2} \mid-1\right)+$
$P\left(r_{1} l_{2}\right.$ or $\left.l_{1} r_{2} \mid+{ }_{1}\right)-1$
P (positional perseveration)
B (position bias)
$=\mathrm{P}\left(\mathrm{r}_{2} \mid \mathrm{r}_{1}\right)+\mathrm{P}\left(\mathrm{l}_{2} \mid \mathrm{l}_{1}\right)-1$
$=2 P\left(r_{2}\right)-1$ if $P\left(r_{2}\right)>P\left(l_{2}\right)$
$2 P\left(l_{2}\right)-1$ if $P\left(l_{2}\right)>P\left(r_{2}\right)$
$\mathbf{R}$ (random)
$=1-D-G-P-B$
$K$ (bias-free performance measure) $=D /(D+R)$

Note $-r$ and $l$ indicate right or left response. + and - indicate correct or incorrect response. Subscripts of 1 and 2 indicate first or second trial of the two-trial sequence. 
The most commonly reported systematic error in animal learning and memory is the simple position habit expressed by significantly more responses to one position than to the other. The state associated with this bias was designated as Position Preference B. If more right than left responses occurred in Trial 2, the proportion of biased responses $\mathbf{P}(b)=\mathbf{P}\left(r_{2}\right) . \mathbf{P}\left(\mathrm{l}_{2}\right)$ was used if left responses predominated. It then followed that $B=2 P(b)-1$.

Another type of position bias occurs if a subject expresses a preference for the position chosen on the previous trial independently of whether that choice was to the right or to the left, or whether it was correct or incorrect. The corresponding state was designated Positional Perseveration P, and the associated responses were perseverated right responses, $r_{2} r_{1}$, or perseverated left responses, $l_{2} I_{1}$. The observed proportion of perseverative responses, $P(p)$, was the unweighted mean of the proportions of right and left perseverative responses. Thus, $P(p)=\left[P\left(r_{2} \mid r_{1}\right)+P\left(l_{2} \mid I_{1}\right)\right] / 2$ and $P=2 P(p)-1$.

When the proportions of responses associated with the preceding states are summed, the total will typically be less than 1 . The difference is the result of the combined effects of unsystematic responding as well as systematic responding not accounted for by the measured states. Possible sources of unaccounted-for systematic responding are response dependencies that extend to trials preceding the previous trial. However, our unpublished analysis of capuchin monkey discrimination and delayed-response data showed that intertrial sequential dependencies diminished to negligible strengths for trials beyond the preceding one. Therefore, we believe that this residual represents the strength of the monkeys' relatively unsystematic responding.

This residual responding, designated by State $\mathbf{R}$ (Random), is defined as the proportion of responses remaining after those attributable to other states have been subtracted. Therefore, $R=1-D$ $-\mathbf{G}-\mathbf{B}-\mathbf{P} . \mathbf{R}$ is best interpreted as the proportion of responses remaining after responses attributable to the strongest systematic sources have been subtracted. $\mathbf{R}$ is certainly not random responding in a profound mathematical sense. However, $R$ should not be regarded as simply a residual category of systematic error that produces responses with the same basic properties as $G, B$, and $P$ since, as we will demonstrate, when delay intervals increase, the strength of $R$ varies differently from that of $G, B$, and $P$.

Occasionally, a monkey displayed a position alternation tendency instead of position perseveration. In such cases, $P$ was negative and the definition of $R$ was modified to include the absolute value of $\mathbf{P}$. Consequently, $\mathbf{R}$ was defined as $\mathbf{1}-\mathbf{D}-\mathbf{G}-\mathbf{B}-|\mathbf{P}|$.

Finally, performance is expressed in terms of a statistic $K$ that reflects correct responding independent of the influence of all systematic errors, namely those attributable to states $G, P$, and $B$. K is defined as $D /(D+R)$. $K$ is an adjusted value of $D$ for which the response population consists of those trials not attributable to systematic errors. Therefore, $\mathrm{K}$ may be regarded as a bias-free performance measure. The use of $\mathrm{K}$ is similar to the use of bias-free measures with classical signal-detection theory (Green \& Swets, 1974) to measure observer sensitivity independent of response bias. More specifically, $\mathbf{K}$ is analogous to the measures $\mathbf{A}^{\prime}$ and $\mathbf{A}$ used as nonparametric sensitivity measures in signal-detection theory (Green, 1964; Grier, 1971). A' and A are estimates of the proportion of correct responses that would occur in the absence of a simple response bias favoring one response (e.g., "yes") over the alternative (e.g., "no"). A measure that directly estimates the proportion of correct responses in the absence of systematic errors is $\mathbf{K}^{\prime}$, defined as $(K+1) / 2 . K$ and $K^{\prime}$ are thus bias-free analogues of $\mathbf{D}$ and $P(+)$, respectively. A difference between $K^{\prime}$ and $A^{\prime}$ or $A$ is that the use of $K^{\prime}$ allows for separation of response sensitivity from sequentially dependent biases (viz., $G$ and $P$ in this experiment) as well as from sequentially independent biases (viz., B), whereas the use of $A^{\prime}$ or $A$ allows for separation of response sensitivity from only one sequentially independent bias.

\section{RESULTS}

Figure 1 depicts the proportion of correct responses for young and aged squirrel monkeys across different delay intervals in Phase 1. Values for $\mathrm{K}^{\prime}$, the estimated proportion of correct responses in the absence of systematic errors, are also displayed in Figure 1. Performance by the young monkeys significantly exceeded that of the aged monkeys in terms of the proportion of correct responses $[F(1,11)=14.16, p=.0029]$ and in terms of $K^{\prime}[F(1,11)$ $=7.55, p=.01891$. Proportion of correct responses and $\mathrm{K}^{\prime}$ both decreased significantly with increasing delays $[F(3,33)=39.02, p<.0001$, and $F(3,33)=13.45$, $p<.0001$, respectively]. However, the age $\times$ delay interaction was not significant for either variable.

Figure 2 depicts the proportion of correct responses and $\mathrm{K}^{\prime}$ during Phase 2 . The young monkeys' performance again exceeded that of the aged monkeys' on both measures $[F(1,10)=6.75, p=.0275$, for proportion of correct responses; $F(1,10)=5.72, p=.0379$, for $\left.\mathrm{K}^{\prime}\right]$. The continuous and true $\mathbf{0}$-delay conditions yielded virtually identical results, but performance dropped abruptly during the 8-sec delay, which resulted in a significant conditions effect for proportion of correct responses $[F(2,20)$ $=41.92, p<.0001]$ and for $\mathrm{K}^{\prime}[F(2,20)=26.70, p<$ $.0001]$. As in Phase 1, the interaction of age and delay conditions did not approach significance for either measure. These interactions were likewise not significant when data for only the true 0 - and 8-sec delays were analyzed.

In Phase 1, the proportion of correct responses following correct responses was slightly but significantly higher than was the proportion following incorrect responses $[64.1$ vs. $62.6, F(1,11)=80.83, p<.0001]$. The corresponding difference in Phase 2 was not significant. The differences were also independent of age.

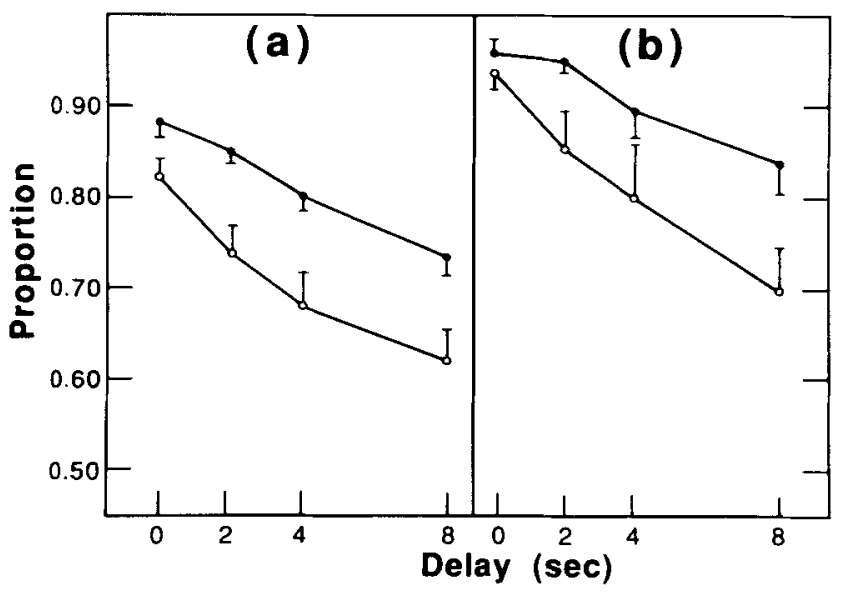

Figure 1. Performance of young (solid circles) and aged (open circles) monkeys in Phase 1. (a) Proportion of correct responses. (b) $\mathrm{K}^{\prime}$ values, which are the estimated proportion of correct responses in the absence of systematic errors. Vertical lines represent 1 SEM. 


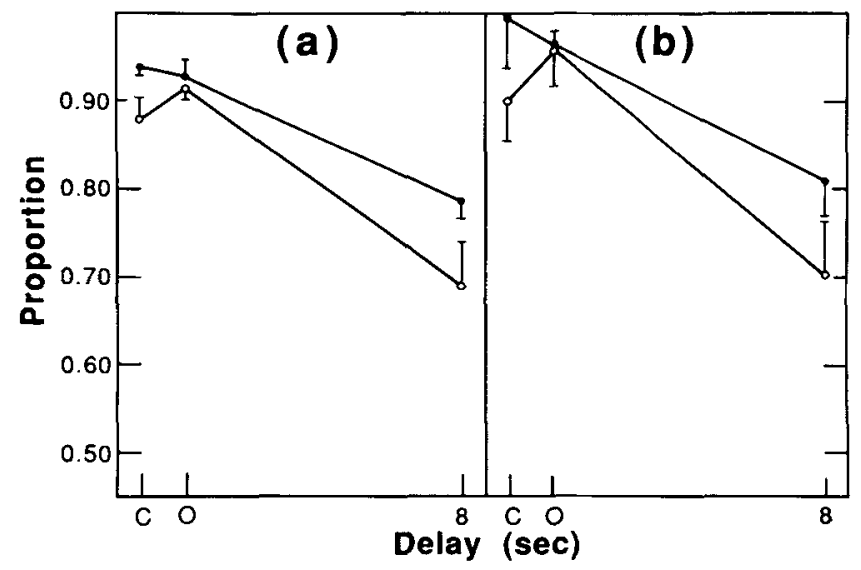

Figure 2. Performance of young (solid circles) and aged (open circles) monkeys in Phase 2. "C" refers to the constant condition and "0" refers to the true 0-sec delay. (a) Proportion of correct responses. (b) $\mathrm{K}^{\prime}$ values, which are the estimated proportion of correct responses in the absence of systematic errors. Vertical lines represent 1 SEM.

The strengths of systematic and nonsystematic error sources can be expressed in two ways. The first measure is as the proportion of all responses attributable to each state. The previously noted formulas express these proportions. The second type of measure expresses proportion of all errors attributable to each state. Proportion of errors attributable to a state is defined as the original state strength divided by $1-D$. This divisor is the proportion of all responses attributable to all error-producing states. If the relative strengths of all error-producing states remain constant as the overall error rate decreases, these proportions will remain constant. Therefore, the proportion of errors attributable to each error source is a measure of the relative importance of systematic and unsystematic errors as overall proportions of errors vary. Table 2 depicts the strengths of all error-producing sources as the proportion of all responses and as the proportion of all errors for both Phase 1 and Phase 2.
In Phase 1, as delays increased, there were significant increases in responses attributable to random responding $[F(3,33)=10.46, p<.0001]$. However, increases in responses attributable to the three sources of systematic errors were not significant. When error strengths were described as the proportion of all errors, the proportion of errors attributable to positional perseveration decreased significantly $[F(3,33)=3.12, p=.0393]$, while the proportion attributable to other error sources did not vary significantly.

To determine the effect of predelay cue removal on the monkeys' proportional allocation of their responses to the different error-producing states, combined data for the $0-, 2-, 4-$, and 8-sec delays were compared with data from the no-cue condition. The combined data were expressed as the proportion of errors attributable to error sources. Therefore, the summed strengths of all error sources were approximately equal in all compared conditions independent of the overall proportion of correct responses; only the relative strengths of the different error sources varied. Removal of the predelay cue resulted in a dramatic increase in the proportion of errors attributable to random responding, from $34.9 \%$ to $60.5 \%[F(1,11)=20.28$, $p=.0009]$. Corresponding significant decreases occurred in the proportion of errors attributable to positional win-shift/lose-stay [17.9\% to $9.0 \% ; F(1,11)=25.27$, $p=.0004]$ and positional perseveration $[19.8 \%$ to $8.7 \%$; $F(1,11)=5.99, p=.0324]$. None of these differences interacted significantly with age. Finally, young monkeys committed a higher proportion of their errors as a result of win-shift/lose-stay responses than did aged monkeys in the cued-delay conditions $[F(1,11)=24.63$, $p=.0004]$.

During Phase 2, increasing delays resulted in significant increases in responses attributable to random responding $[F(2,20)=20.17, p<.0001]$, positional win-shift/ lose-stay $[F(2,20)=3.72, p=.0422]$, and position preference $[F(2,20)=10.56, p=.0007]$. The aged monkeys committed more random errors than did the young

Table 2

Proportion of All Responses and Proportion of All Errors Attributable to Different Error-Producing Sources in Phases 1 and 2

Phase 1

\begin{tabular}{|c|c|c|c|c|c|c|c|c|c|}
\hline \multirow[b]{3}{*}{ Error Source } & \multicolumn{9}{|c|}{ Phase 1} \\
\hline & \multicolumn{4}{|c|}{ All Responses } & \multicolumn{5}{|c|}{ All Errors } \\
\hline & 0 & 2 & 4 & 8 & NC & $\mathbf{0}$ & 2 & 4 & 8 \\
\hline Positional win-shift/lose-stay & .02 & .01 & .07 & .04 & .09 & .15 & .20 & .21 & .14 \\
\hline Position habit & .09 & .11 & .14 & .15 & .16 & .28 & .24 & .28 & .23 \\
\hline Position perseveration & .08 & .10 & .05 & .08 & .09 & .29 & .26 & .13 & .15 \\
\hline Random & .06 & .12 & .20 & .29 & .60 & .28 & .31 & .38 & .44 \\
\hline
\end{tabular}

Phase 2

Positional win-shift/lose-stay Position habit Position perseveration Random

\begin{tabular}{|c|c|c|c|c|c|}
\hline \multicolumn{3}{|c|}{ All Responses } & \multicolumn{3}{|c|}{ All Errors } \\
\hline C & $\mathbf{0}$ & 8 & C & 0 & 8 \\
\hline .09 & .06 & .21 & .26 & .23 & .26 \\
\hline .04 & .05 & .12 & .25 & .41 & .24 \\
\hline .05 & .03 & .02 & .40 & .33 & .09 \\
\hline .14 & .10 & .54 & .32 & .33 & .63 \\
\hline
\end{tabular}

Note $-0,2,4$, and 8 refer to delay (in seconds) between offset of the window light and presentation of the reward; "NC" refers to the no-cue condition; "C" refers to the constant condition. 
monkeys $[F(1,10)=5.21, p=.0475]$, but did not differ from the young monkeys in frequency of any nonrandom error source.

When state strengths were expressed as proportions of total errors, random responding again increased significantly with increasing delays $[F(2,20)=9.27, p=$ $.0002]$. Changes in the individual systematic errors were not significant. None of these proportions varied significantly with age.

\section{DISCUSSION}

The aged squirrel monkeys committed more errors than did the young adult squirrel monkeys in both phases of this experiment. The details of this difference support two conclusions. First, the age-related deficit was not a result of the aged monkeys being particularly susceptible to systematic error production; systematic errors did not vary with age in either phase. Moreover, in Phase 2 the aged monkeys committed more random errors than did the young monkeys but did not differ in systematic error production. In addition, when performance was defined independent of systematic errors by $\mathrm{K}^{\prime}$, age-related differences over delays paralleled differences observed when performance was defined as the proportion of correct responses. If aging had resulted in an increased likelihood of committing systematic errors, the age-related difference in $\mathrm{K}^{\prime}$ would have been less than the difference in the proportion of correct responses. Thus, on two-choice delayed-response problems, poorer performance by aged monkeys was not necessarily accompanied by increased perseverative and other systematic errors.

The second conclusion is that the age-related increases in errors did not provide evidence of a memory deficit. The age $\times$ delay interaction was not significant in either phase. If memory for the predelay cue had been affected by aging, the age-related deficit would have been greatest at the longest delays and the interaction would have been significant (see Bartus et al., 1980; Medin, 1969). Riopelle and Rogers (1965) reported a similar lack of an age $x$ delay interaction in chimpanzees on a five-choice delayed response. Their aged chimpanzees were about equally deficient in all delay conditions, including 0 -sec delays, a result that the authors attributed to attentional deterioration in the aged animals. A similar conclusion may apply to the aged squirrel monkeys in the present experiment. The absence of a delay $x$ conditions interaction when performance after relatively long delays was compared with that requiring virtually no memory (viz., the constant and true 0 conditions in Phase 2 and the 0 -sec condition in Phase 1) suggests that the aged monkeys simply did not attend to the predelay cue light as effectively as did the young monkeys. This result may be related to the greater distractibility of aged squirrel monkeys in a simple operant task (Harrison \& Isaac, 1984).

The lack of an age-related memory deficit is not inconsistent with previous reports of age-related memory deficits in delayed response by rhesus (Bartus et al.,
1978; Medin, 1969) and capuchin (Bartus et al., 1980) monkeys. In these studies, the correct response locations were cells within $3 \times 3$ or $4 \times 4$ matrices. Therefore, correct responding probably necessitated considerable spatial memory. Considerable evidence indicates the sensitivity of spatial memory to normal aging in humans (Flicker, Bartus, Crook, \& Ferris, 1984; Park, Puglisi, \& Sovacool, 1983) and in rats (Barnes \& McNaughton, 1985), although some disagreement still exists (e.g., Meudell, 1983). Unequivocal spatial memory enables the subject to remember a location based only on the relationship of that location to its surrounding environment. In contrast, the correct location in a two-choice delayedresponse problem can be remembered on the basis of both overt and covert nonspatial strategies. An example of the former is maintenance of a bodily orientation toward the correct location, a behavior that squirrel monkeys frequently display, despite their high activity level (French, 1959). An example of a covert strategy is the retention of a kinesthetic-based tendency to respond to the right or to the left.

During the cued-delay conditions in Phase 1, the young monkeys committed a higher proportion of their errors as a result of positional win-shift/lose-stay responses than did the aged monkeys. This result could be interpreted as a subtle indication of a memory deficit in the aged monkeys. Positional win-shift/lose-stay errors were based on memory of the location chosen about $25 \mathrm{sec}$ earlier (on the preceding trial). Consequently, if the aged monkeys were less able to remember the previously chosen location, they would have committed fewer errors of this type. The age difference may not have been apparent for the simple proportional strengths of State F simply because the greater overall number of errors by the aged monkeys may have masked the proportional difference. However, whether or not this memory hypothesis is correct, the age difference in the proportion of win-shift/ lose-stay errors further emphasizes the resistance of aged monkeys to systematic errors.

In both phases, the proportion of all errors attributable to unsystematic errors generally increased, and the proportion attributable to systematic errors decreased, as delay interval increased. The disproportionately high increase in unsystematic responding was particularly enhanced during the no-cue trials in Phase 1. Thus, as uncertainty about the correct response was increased by longer delays or by complete removal of the predelay cue, the contribution of unsystematic responding to the extra error output increased. Furthermore, the proportional increase in unsystematic responding was equally strong in young and aged monkeys in spite of the greater number of errors committed by the aged monkeys.

In the past, the nonsystematic aspect of animal responding was regarded as a part of the residual error or as the inevitable noise in any behavioral-response system. Unsystematic responses, even more than systematic errorproducing responses, were deemed uninteresting and were therefore virtually ignored. However, an effective strategy 
displayed in an enormously wide variety of biological adaptions is one in which the organism (or species) generates a diverse set of responses in the face of uncertainty (Changeux, Heidmann, \& Patte, 1984; Skinner, 1984). A selection process then strengthens the more effective responses. In a learning or memory task, unsystematic responding could be regarded as the strategy of the ideal responder when confronted with highly uncertain response-outcome rules (assuming that the ideal responder is not capable of generating and testing hypotheses). For example, if an animal displays a constant position habit on a problem in which position is an irrelevant dimension, correct responding will inevitably remain at chance level. However, as responding becomes increasingly unsystematic, above-chance performance becomes increasingly likely to occur and to be reinforced. At this point, selection (i.e., the law of effect) can strengthen the effective behavior. This mechanism is primitive but effective. The present experiment indicates that the generator of response diversity in squirrel monkeys is robust and does not deteriorate with old age, at least in simple delayed-response problems that do not provoke high levels of response bias. It remains to be demonstrated whether the random-dominated response to uncertainty diminishes in aged squirrel monkeys confronted with problems rich in sources of systematic response biases.

\section{REFERENCES}

Barnes, C. A., McNaughton, B. L. (1985). Spatial information: How and where is it stored? In N. M. Weinberger, J. L. McGaugh, \& G. Lynch (Eds.), Memory systems of the brain (pp. 49-61). New York: Guilford.

Bartus, R. T., Dean, R. L., \& Beer, B. (1980). Memory deficits in aged cebus monkeys and facilitation with central cholinomimetics. Neurobiology of Aging, 1, 145-152.

Bartus, R. T., Dean, R. L., \& Fleming, D. L. (1979). Aging in the rhesus monkey: Effects on visual discrimination and reversal learning. Journal of Gerontology, 34, 209-219.

Bartus, R. T., Fleming, D. L., \& Johnson, H. R. (1978). Aging in the rhesus monkey: Debilitating effects on short-term memory. Journal of Gerontology, 33, 858-871.

Bernstein, I. S. (1961). Response variability and rigidity in the adult chimpanzee. Journal of Gerontology, 16, 381-386.

BIRREN, J. E. (1962). Age differences in learning a two-choice water maze by rats. Journal of Gerontology, 17, 207-213.

Changeux, J. P., Heidmann, T., \& Patte, P. (1984). Learning by selection. In P. Marler \& H. S. Terrace (Eds.), The biology of learning (pp. 115-133). Berlin: Springer-Verlag.

Elias, P. K., \& Elias, M. F. (1976). Effects of age on leaming ability: Contributions from the animal literature. Experimental Aging Research, 2, 165-186.

Flicker, C., Bartus, R. T., Crook, T. H., \& Ferris, S. H. (1984).
Effects of aging and dementia upon recent visuospatial memory. Psychobiology of Aging, 5, 275-283.

FoBES, J. L., KING, J. E. (1982). Measuring primate learning abilities. In J. L. Fobes \& J. E. King (Eds.), Primate behavior (pp. 289 326). New York: Academic Press.

FrenCH, G. M. (1959). Performance of squirrel monkeys on variants of delayed response. Journal of Comparative \& Physiological Psychology, 52, 741-745.

GoodrICK, C. L. (1968). Learning, retention, and extinction of a complex maze habit for mature-young and senescent Wistar albino rats. Journal of Gerontology, 23, 298-304.

Gooprick, C. L. (1972). Learning by mature-young and aged Wistar albino rats as a function of test complexity. Journal of Gerontology, 27, 353-357.

GooDRICK, C. L. (1975). Behavioral rigidity as a mechanism for facilitation of problem solving for aged rats. Joumal of Gerontology, 30 , 181-184.

GrEeN, D. M. (1964). General prediction relating yes-no and forced choice results. Journal of the Acoustical Society of America, 36, 1042

GREEN, D. M., \& SweTs, J. A. (1974). Signal detection and theory and psychophysics. New York: Krieger.

GRIER, J. B. (1971). Nonparametric indices for sensitivity and bias: Computing formulas. Psychological Bulletin, 75, 424-429.

HarLow, H. F. (1959). Learning set and error factor theory. In S. Koch (Ed.), Psychology: A study of a science (Vol. 2, pp. 492-537). New York: McGraw-Hill.

HARRISON, D. W., \& ISAAC, W. (1984). Disruption and habituation of stable fixed-interval behavior in younger and older monkeys. Physiology \& Behavior, 32, 341-344.

Hopf, S., \& PlOOG, D. (1979). Life span in captive squirrel monkeys (Saimiri) with pathological and reproductive records. Primates, 20 , 313-316.

Kubanis, P., \& Zornetzer, S. F. (1981). Age related behavioral and neurobiological changes: A review with an emphasis on memory. Behavioral \& Neural Biology, 31, 115-172.

LENTZ, J. L., \& KING, J. E. (1981). Sources of errors by capuchin monkeys on delayed response. Animal Learning \& Behavior, 9, 183-188.

Levine, M. (1965). Hypothesis behavior. In A. M. Schrier, H. F. Harlow, \& F. Stollnitz (Eds.), Behavior of nonhuman primates (Vol. 1, pp. 97-127). New York: Academic Press.

MEDIN, D. L. (1969). Form perception and pattern reproduction by monkeys. Joumal of Comparative \& Physiological Psychology, 68 412-419.

Meudell, P. R. (1983). The development and dissolution of memory. In A. Mayes (Ed.), Memory in humans and animals (pp. 83-132). New York: Van Nostrand Reingold.

Park, P. C., Puglisi, J. T., \& Sovacool, J. (1983). Memory for pictures, words, and spatial location in older adults: Evidence for pictorial superiority. Joumal of Gerontology, 38, 582-588.

Riopelle, A. J., \& Rogers, C. M. (1965). Age changes in chimpanzees. In A. M. Schrier, H. F. Harlow, \& F. Stollnitz (Eds.), Behavior of nonhuman primates (Vol. 2, pp. 449-462). New York: Academic Press.

SkINNER, B. F. (1984). Selection by consequences. Behavioral \& Brain Sciences, 7, 477-510.

(Manuscript received June 28, 1988; revision accepted for publication November 22,1988 .) 